\title{
UNITARY REPRESENTATIONS OF LIE GROUPS AND GÅRDING'S INEQUALITY
}

\author{
OLA BRATTELI, FRED M. GOODMAN, \\ PALLE E. T. JØRGENSEN AND DEREK W. ROBINSON
}

(Communicated by Paul S. Muhly)

\begin{abstract}
We prove two versions of Gårding's inequality for strongly elliptic operators in the enveloping Lie algebra associated with a unitary representation of a Lie group. We then deduce a characterization of the differential structure of the representation in terms of the elliptic operators.
\end{abstract}

\section{INTRODUCTION}

Let $(\mathscr{H}, G, U)$ denote a continuous representation of the connected Lie group $G$ by unitary operators $U(g), g \in G$, on the Hilbert space $\mathscr{H}$. Fix a basis $a_{1}, \ldots, a_{d}$ of the Lie algebra $\mathfrak{g}$ of $G$ and let $A_{1}, \ldots, A_{d}$ denote the skew self-adjoint generators of the one-parameter subgroups $t \in \mathbb{R} \mapsto U\left(e^{-t a_{i}}\right)$. If $\alpha=\left(\alpha_{1}, \ldots, \alpha_{d}\right)$, with $\alpha_{i} \geq 0$ and $|\alpha|=\alpha_{1}+\cdots+\alpha_{d}$, we define $A^{\alpha}=$ $A_{1}^{\alpha_{1}} A_{2}^{\alpha_{2}} \cdots A_{d}^{\alpha_{d}}$ and set $\mathscr{H}_{\infty}=\bigcap_{\alpha} D\left(A^{\alpha}\right)$. It follows by standard reasoning that $\mathscr{H}_{\infty}$ is norm dense in $\mathscr{H}^{\infty}$.

A form

$$
\xi \in \mathbb{R}^{d} \rightarrow C_{m}(\xi)=\sum_{\alpha ;|\alpha| \leq m} c_{\alpha} \xi^{\alpha} \in \mathbb{C},
$$

where $\xi^{\alpha}=\xi_{1}^{\alpha_{1}} \xi_{2}^{\alpha_{2}} \ldots \xi_{d}^{\alpha_{d}}$, is defined to be strongly elliptic if $\operatorname{Re}\left((-1)^{m / 2} P_{m}(\xi)\right)$ $>0$ for all $\xi \in \mathbb{R}^{d} \backslash\{0\}$, where $P_{m}$ denotes the principal part of $C_{m}$, i.e.,

$$
P_{m}=\sum_{\alpha ;|\alpha|=m} c_{\alpha} \xi^{\alpha} .
$$

Equivalently, $C_{m}$ is strongly elliptic if there is a $p>0$ such that

$$
\operatorname{Re}\left((-1)^{m / 2} P_{m}(\xi)\right) \geq p|\xi|^{m}
$$

for all $\xi \in \mathbb{R}^{d}$. The largest value $p_{m}\left(=p_{m}(c)\right)$ of $p$ for which this estimate is valid is called the ellipticity constant of $C_{m}$. Note that the strong ellipticity condition implies automatically that $m$ is even.

Received by the editors June 17, 1988.

1980 Mathematics Subject Classification (1985 Revision). Primary 47D40, 47F05, 22E30; Secondary $47 \mathrm{H} 06,47 \mathrm{~B} 25$. 
Next we associate with each unitary representation $(\mathscr{H}, G, U)$, each basis $a_{1}, \ldots, a_{d}$, of $\mathfrak{g}$, and each strongly elliptic form $C_{m}$, an operator

$$
A_{m}(c)=\sum_{\alpha ;|\alpha| \leq m} c_{\alpha} A^{\alpha}
$$

with domain $D\left(A_{m}\right)=\mathscr{H}_{\infty}$. We refer to the $A_{m}$ as strongly elliptic operators. The simplest example is the Laplacian

$$
\Delta=-\sum_{i=1}^{d} A_{i}^{2}
$$

corresponding to the form $\xi \mapsto-|\xi|^{2}$.

Theorem 1.1. Let $A_{m}(c)$ be strongly elliptic. For each $p \in\left\langle 0, p_{m}(c)\right\rangle$ there is a $q \geq 0$ such that

$$
\operatorname{Re}\left(x, A_{m}(c) x\right) \geq p\left(x, \Delta^{m / 2} x\right)-q(x, x)
$$

for all $x \in \mathscr{H}_{\infty}$. Moreover, $q$ can be chosen independently of the particular unitary representation.

An alternative, weaker version of the theorem can be stated in terms of the $C^{n}$-norms of the representation. These are defined by $\|x\|_{0}=\|x\|$ and

$$
\|x\|_{n}=\sup _{0 \leq i \leq d}\left\|A_{i} x\right\|_{n-1}
$$

where $A_{0}=I$. The $C^{n}$-subspace

$$
\mathscr{H}_{n}=\bigcap_{\alpha ;|\alpha| \leq n} D\left(A^{\alpha}\right)
$$

is a Banach space with respect to $\|\cdot\|_{n}$, and $\mathscr{H}_{\infty}$ is $\|\cdot\|_{n}$-dense in $\mathscr{H}_{n}$ (see, for example, [G]).

Theorem 1.2. Let $A_{m}(c)$ be strongly elliptic. For each $p^{\prime} \in\left\langle 0, p_{m}(c)\right\rangle$ there is a $q^{\prime} \geq 0$ such that

$$
\operatorname{Re}\left(x, A_{m}(c) x\right) \geq p^{\prime}\|x\|_{m / 2}^{2}-q^{\prime}\|x\|^{2}
$$

for all $x \in \mathscr{H}_{\infty}$. Moreover, $q$ can be chosen independently of the particular unitary representation.

It was established by R. Goodman [G] that $\mathscr{H}_{n}=D\left(\Delta^{n / 2}\right)$ and the norm $\|\cdot\|_{n}$ on $\mathscr{H}_{n}$ is equivalent to the graph norm $x \rightarrow\left\|\Delta^{n / 2} x\right\|+\|x\|$. But more recently ([R1], [ $R 2$ ]) the following more precise estimates have been obtained; for each $n=1,2, \ldots$ and $\epsilon>0$ there is a $c_{n}(\epsilon)$ such that

$$
\|x\|_{n} \leq(1+\epsilon)\left\|\Delta^{n / 2} x\right\|+c_{n}(\epsilon)\|x\|
$$

for all $x \in \mathscr{H}_{n}$. Therefore Theorem 1.2 follows easily from Theorem 1.1. Next we sketch the proof of the latter result. It is essentially a consequence of the work of Langlands [ $L 1$ ], [ $L 2$ ]. 


\section{OUtLINE OF THE PROOF OF THEOREM 1.1}

If $C_{m}$ is a strongly elliptic form with ellipticity constant $p_{m}$ and $p \in\left\langle 0, p_{m}\right\rangle$ then the form $C_{m}^{\prime}$ defined by

$$
C_{m}^{\prime}(\xi)=C_{m}(\xi)-p\left(-|\xi|^{2}\right)^{m / 2}
$$

is also strongly elliptic, with ellipticity constant $p_{m}^{\prime}=p_{m}-p$. Since $A_{m}\left(c^{\prime}\right)=$ $A_{m}(c)-p \Delta^{m / 2}$ the inequality (1.1) is equivalent to the lower semiboundedness property

$$
\operatorname{Re}\left(x, A_{m}\left(c^{\prime}\right) x\right) \geq-q(x, x) .
$$

Therefore to prove Theorem 1.1 it suffices to prove that the real part of every strongly elliptic operator $A_{m}(c)$ is lower semibounded.

Let $A_{m}^{\dagger}$ denote the formal adjoint of $A_{m}$ on $\mathscr{H}_{\infty}$, i.e.,

$$
A_{m}^{\dagger}=\sum_{\alpha ;|\alpha| \leq m} \bar{c}_{\alpha}(-1)^{|\alpha|} A^{\alpha *}
$$

on $\mathscr{H}_{\infty}$ where $A^{\alpha_{*}}=A_{d}^{\alpha_{d}} \cdots A_{1}^{\alpha_{1}}$. Then $R_{m}=\left(A_{m}+A_{m}^{\dagger}\right) / 2$ is a symmetric operator on $\mathscr{H}_{\infty}$. But it follows from the structure relations of $\mathfrak{g}$ that $A^{\alpha_{*}}=A^{\alpha}$ modulo lower order terms, i.e.,

$$
A^{\alpha *}=A^{\alpha}+\sum_{\beta ;|\beta|<|\alpha|} c_{\alpha, \beta} A^{\beta},
$$

where the $c_{\alpha, \beta}$ are polynomials in the structure constants. Therefore $R_{m}$ is a strongly elliptic operator associated with a form $C_{m}^{\prime}$ whose principal part $P_{m}^{\prime}$ is given by

$$
P_{m}^{\prime}(\xi)=\sum_{\alpha ;|\alpha|=m}\left(\operatorname{Re} c_{\alpha}\right) \xi^{\alpha}
$$

Now it follows from Langlands' [ $L 1$ ] first theorem that $R_{m}$ is essentially selfadjoint, and from his second theorem that the self-adjoint closure $\bar{R}_{m}$ generates a continuous semigroup, which is automatically self-adjoint. But then $\bar{R}_{m}$ is lower semibounded by spectral theory. Hence there is a $q \geq 0$ such that

$$
\operatorname{Re}\left(x, A_{m} x\right)=\left(x, R_{m} x\right) \geq-q(x, x)
$$

for all $x \in \mathscr{H}_{\infty}$. Therefore (1.1) follows from the previous reasoning.

Finally, Langlands' $[L 1]$ third theorem establishes that the semigroup $S$ generated by $\bar{R}_{m}$ has a representation independent kernel, i.e.,

$$
S_{t}=\int_{G} d g p_{t}(g) U(g),
$$

where $d g$ denotes the left invariant Haar measure and $p_{t} \in L_{1}(G ; d g)$. Since

$$
e^{q t} \leq \int_{G} d g\left|p_{t}(g)\right|
$$


one can then choose $q$ to be independent of the particular unitary representation. This completes the outline of the proof.

\section{Differential Structure}

Let $A_{m}=A_{m}(c)$ be a strongly elliptic operator with formal adjoint $A_{m}^{\dagger}$ and define

$$
B_{2 m}=A_{m}^{\dagger} A_{m}=\sum_{\alpha ;|\alpha| \leq m} \sum_{\beta ;|\beta| \leq m} \bar{c}_{\alpha} c_{\beta}(-1)^{|\alpha|} A^{\alpha_{*}} A^{\beta}
$$

on $\mathscr{H}_{\infty}$. Since $A^{\alpha_{*}} A^{\beta}=A^{\alpha+\beta}$ modulo lower-order terms, it follows that $B_{2 m}$ is a strongly elliptic operator. Moreover, if $P_{m}$ denotes the principal part of the form $C_{m}$ associated with $A_{m}$ and $P_{2 m}^{\prime}$ the principal part of the form $C_{2 m}^{\prime}$ associated with $B_{2 m}$ then

$$
P_{2 m}^{\prime}(\xi)=\left|P_{m}(\xi)\right|^{2} \geq\left(\operatorname{Re} P_{m}(\xi)\right)^{2} .
$$

Therefore one has the inequality $p_{2 m}^{\prime} \geq p_{m}^{2}$ for the ellipticity constants, with equality whenever the principal part of $C_{m}$ is real. Now applying Theorem 1.2 to $B_{2 m}$ one deduces the following.

Corollary 3.1. Let $A_{m}(c)$ be strongly elliptic. For each $p \in\left\langle 0, p_{m}(c)\right\rangle$ there is a $q \geq 0$, independent of the representation, such that

$$
\|x\|_{m} \leq(1 / p)\left\|A_{m}(c) x\right\|+q\|x\|
$$

for all $x \in \mathscr{H}_{\infty}$. Consequently $A_{m}(c)$ is closed on $\mathscr{H}_{m}$ and the $C^{m}$-norm $\|\cdot\|_{m}$ is equivalent to the graph $x \mapsto\left\|A_{m}(c) x\right\|+\|x\|$.

Proof. Replacing $A_{m}(c)$ by $B_{2 m}=A_{m}(c)^{\dagger} A_{m}(c)$ in (1.2) one finds for each $p^{\prime} \in\left\langle 0, p_{2 m}^{\prime}\right\rangle$ a $q^{\prime} \geq 0$ such that

$$
p^{\prime}\|x\|_{m}^{2} \leq\left\|A_{m}(c) x\right\|^{2}+q^{\prime}\|x\|^{2}
$$

for all $x \in \mathscr{H}_{\infty}$. But $p_{2 m}^{\prime} \geq p_{m}^{2}$. Thus if $p \in\left\langle 0, p_{m}\right\rangle$ and $p^{\prime}=p^{2}$,

$$
\|x\|_{m}^{2} \leq\left(1 / p^{2}\right)\left\|A_{m}(c) x\right\|^{2}+q^{\prime}\|x\|^{2} .
$$

Then (3.1) follows by elementary reasoning. But

$$
\left\|A_{m}(c) x\right\| \leq\left[\sum_{\alpha ;|\alpha| \leq m}\left|c_{\alpha}\right|\right]\|x\|_{m}
$$

and since $\mathscr{H}_{\infty}$ is $\|\cdot\|_{m}$-dense in $\mathscr{H}_{m}$ one immediately deduces the last statement of the corollary from (3.1) and (3.2).

Finally the foregoing reasoning extends to higher-order products. If $A_{m_{1}}, \ldots$, $A_{m_{n}}$ are all strongly elliptic and $m=m_{1}+\cdots+m_{n}$ then

$$
B_{2 m}=\left(A_{m_{n}}^{\dagger} \cdots A_{m_{1}}^{\dagger}\right)\left(A_{m_{1}} \cdots A_{m_{n}}\right)
$$


is a strongly elliptic operator of order $2 m$. Hence the same arguments show that the $C^{m}$-norm is equivalent to the graph norm

$$
x \mapsto\left\|A_{m_{1}} \cdots A_{m_{n}} x\right\|+\|x\| .
$$

Detailed proofs of these results will appear in [R2].

\section{Semigroups Bounds}

The closure $\bar{A}_{m}$ of each strongly elliptic operator $A_{m}$ generates a strongly continuous semigroup $S$ holomorphic in a sector $\Delta_{m}(\varphi)=\{z \in \mathbf{C} ; \operatorname{Re} z>0$, $|\operatorname{Arg} z|<\varphi\}$ by Langlands' second theorem. Then if $\theta \in[0, \varphi\rangle$ it follows by general theory that there exist $M_{\theta} \geq 1$ and $\omega_{\theta} \geq 0$ such that

$$
\left\|S_{z}\right\| \leq M_{\theta} e^{\omega_{\theta}|z|}
$$

whenever $\operatorname{Re} z \geq 0$ and $|\operatorname{Arg} z| \leq \theta$. But the Gårding inequalities allow one to infer that $M_{\theta}=1$, at least for small $\theta$.

Corollary 4.1. Let $C_{m}$ be a strongly elliptic form with ellipticity constant $p_{m}$, define

$$
q_{m}=\sum_{\alpha ;|\alpha|=m}\left|\operatorname{Im} c_{\alpha}\right|
$$

and $\varphi_{m}=\operatorname{Tan}^{-1} p_{m} / q_{m}$. Further let $S$ denote the holomorphic semigroup generated by $\overline{A_{m}(c)}$.

If $\theta \in\left[0, \varphi_{m}\right)$ then there is an $\omega_{\theta} \geq 0$ such that

$$
\left\|S_{z}\right\| \leq e^{\omega_{\theta}|z|}
$$

for all $z \in \mathbf{C}$ with $\operatorname{Re} z>0$, and $|\operatorname{Arg} z| \leq \theta$.

Proof. First, by Langlands' estimates [L2], or by [R2], the semigroup $S$ is holomorphic in the sector $\Delta_{m}\left(\varphi_{m}\right)$, and possibly in a larger sector. Thus if $z \in \mathbf{C}$ wich $\operatorname{Re} z>0$ and $|\operatorname{Arg} z| \leq \theta$ then $z A_{m}$ generates a continuous semigroup. But

$$
\begin{aligned}
\operatorname{Re}\left(x, z A_{m} x\right)= & (\operatorname{Re} z)\left(\operatorname{Re}\left(x, A_{m} x\right)-(\operatorname{Im} z) \operatorname{Im}\left(x, A_{m} x\right)\right. \\
\geq & (\operatorname{Re} z) \operatorname{Re}\left(x, A_{m} x\right)-|\operatorname{Im} z| q_{m}\|x\|_{m / 2}^{2} \\
& -|\operatorname{Im} z| r_{m}\|x\|_{m / 2} \cdot\|x\|_{m / 2-1},
\end{aligned}
$$

where

$$
r_{m}=\sum_{\alpha ;|\alpha| \leq m}\left|\operatorname{Im} c_{\alpha}\right|
$$

Now for each $\delta>0$,

$$
\|x\|_{m / 2} \cdot\|x\|_{m / 2-1} \leq \delta\|x\|_{m / 2}^{2}+(1 / 4 \delta)\|x\|_{m / 2-1}^{2} .
$$

Moreover, for each $\sigma>0$ there is a $k_{\sigma}>0$ such that

$$
\|x\|_{m / 2-1}^{2} \leq \sigma\|x\|_{m / 2}^{2}+k_{\sigma}\|x\|^{2} .
$$


Hence for each $\varepsilon>0$ there is a $c_{\varepsilon}>0$ such that

$$
\operatorname{Re}\left(x, z A_{m} x\right)=(\operatorname{Re} z) \operatorname{Re}\left(x, A_{m} x\right)-|\operatorname{Im} z|\left(q_{m}+\varepsilon\right)\|x\|_{m / 2}^{2}-|\operatorname{Im} z| c_{\varepsilon}\|x\|^{2} .
$$

Now we can use the second form of Gårding's inequality (1.2) to deduce that for each $p^{\prime} \in\left\langle 0, p_{m}\right\rangle$ there is a $q^{\prime}>0$ such that

$\operatorname{Re}\left(x, z A_{m} x\right) \geq(\operatorname{Re} z) \operatorname{Re}\left(x, A_{m} x\right)\left(1-\frac{|\operatorname{Im} z|}{\operatorname{Re} z} \frac{q_{m}+\varepsilon}{p^{\prime}}\right)-|\operatorname{Im} z| \cdot\|x\|^{2}\left(q_{m} \frac{q^{\prime}}{p^{\prime}}+c_{\varepsilon}\right)$.

But by choosing $p^{\prime}$ close to $p_{m}$ and $\varepsilon$ small, one can assure that $\left(1-\left(q_{m}+\varepsilon\right)|\operatorname{Im} z| / p^{\prime} \operatorname{Re} z\right) \geq 0$. Then by another application of Gårding's inequality there is a $q \geq 0$ such that $\operatorname{Re}\left(x, A_{m} x\right) \geq-q\|x\|^{2}$. Therefore

$$
\operatorname{Re}\left(x,(z /|z|) A_{m} x\right) \geq-\omega_{\theta}\|x\|^{2}
$$

with $\omega_{\theta}=q\left(1-\left(\left(q_{m}+\varepsilon\right) / p^{\prime}\right) \operatorname{Tan} \theta\right)+q^{\prime} q_{m} / p^{\prime}+c_{\varepsilon}$.

Finally,

$$
\frac{d}{d|z|}\left\|S_{z} x\right\|^{2} e^{-2 \omega_{\theta}|z|}=-\operatorname{Re}\left(S_{z} x,\left((z /|z|) A_{m}+\omega_{\theta} I\right) S_{z} x\right) e^{-2 \omega_{\theta}|z|} \leq 0 .
$$

Therefore, by integration,

$$
\left\|S_{z} x\right\| \leq e^{\omega_{\theta}|z|}\|x\|
$$

for all $z \in \mathbb{C}$ with $\operatorname{Re} z \geq 0$ and $|\operatorname{Arg} z| \leq \theta$.

\section{REFERENCES}

[G] R. Goodman, One parameter groups generated by operators in an enveloping algebra, J. Funct. Anal. 6 (1970), 218-236.

[L1] R. P. Langlands, Some holomorphic semigroups, Proc. Nat. Acad. Sci. 46 (1960), 361-363.

[L2] _ Semigroups and representations of Lie groups, Yale University thesis, 1960.

[R1] D. W. Robinson, The differential and integral structure of representation of Lie groups, J. Operator Theory 19 (1988), 95-128.

[R2] _ - The analytic structure of representations of Lie groups (in preparation).

Department of Mathematics, The University of Iowa, Iowa City, lowa 52242

institute of Mathematics, University of Trondheim, N-7034 Trondheim, Norway

Department of Mathematics, Institute of Advanced Studies, Australian National University, CANBerra ACT 2601, Australia 